\title{
Novel Sample Preparation for Operando TEM Studies
}

\author{
B.K. Miller, T.M. Barker, and P.A. Crozier
}

School for Engineering of Matter, Transport and Energy, Arizona State University, Tempe, AZ 852876106

In situ studies of catalysts in the environmental transmission electron microscope (ETEM) are of great value to elucidate the structure of a catalyst under reaction conditions. This is important, since the structure of many catalysts change at elevated temperature in the presence of reactive gases [1-2]. One way to directly relate structure to activity is to measure the activity of the catalyst while it is being observed in the TEM, and this is called operando TEM. We measure catalytic activity by monitoring the amount of product gas from the catalytic reaction using electron energy-loss spectroscopy (EELS) and mass spectrometry [4]. The minute amount of catalyst present in a typically prepared powder TEM sample is usually not enough to produce a detectable amount of product gas. A new sample preparation technique was therefore developed to increase the amount of catalyst present in the microscope by at least an order of magnitude.

The pellet was formed by crushing glass fibers, packing them into a $3 \mathrm{~mm}$ quartz cylinder, and firing them at $680^{\circ} \mathrm{C}$ for $2 \mathrm{hrs}$. Alternatively, quartz fibers may be used and fired at $1150^{\circ} \mathrm{C}$, allowing higher temperature (up to $900^{\circ} \mathrm{C}$ ) and photocatalytic experiments to be performed in-situ, due to quartz's higher melting temperature and transparency in the UV range. The resulting sintered fiber cylinder was cut into slices which were then ground to their final thickness of about $0.5 \mathrm{~mm}$. A $0.5 \mathrm{~mm}$ hole was drilled through the center to allow for imaging, as seen in Figure 2. This pellet provides the high-surface-area structure needed to disperse the $200 \mathrm{~nm}$ silica spheres which are the support we currently use for the ruthenium catalyst; other supports could also be used. The catalyst dispersed on the glass/quartz fibers cannot be imaged at high resolution however, because the insulating fibers in the pellet charge significantly under electron beam irradiation. Thus, a conductive metal mesh with catalyst dispersed over its surface is also placed into the holder before the pellet (Figure 1c).

Many parameters affect the performance of this unique sample configuration. The density/porosity of the pellet, the loading of the catalyst, the size of the central hole, and the materials selected for the metal mesh, and pellet fibers are all important for achieving good results. We will discuss these and other factors, and show data, like that given in figure 3, from operando experiments on $\mathrm{CO}$ oxidation over a $\mathrm{Ru} / \mathrm{RuO}_{2}$ catalyst which used this new sample prep technique.

\section{References:}

[1] P.L. Gai. Topics in Catalysis 8, (1999), p.97-113.

[2] P.L. Gai, et al. MRS Bulletin 32, (2007), p.1044-1050.

[3] B.K. Miller, P.A. Crozier Microscopy and Microanalysis (submitted)

[4] The support from US Department of Energy (DE-SC0004954) and the use of ETEM at John M.

Cowley Center for HR Microscopy at Arizona State University is gratefully acknowledged. 


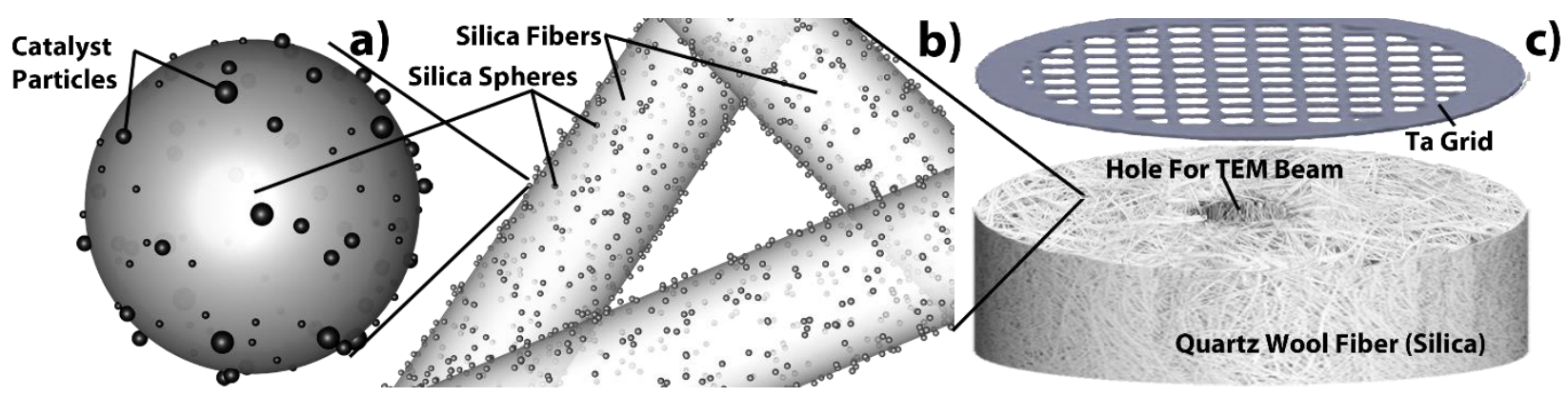

Figure 1. Schematic showing the structure of the TEM sample. a) Silica spheres of about $200 \mathrm{~nm}$ diameter are used as the catalyst support. b) The silica spheres are in turn dispersed over glass wool fibers (also silica), which have been formed into a $3 \mathrm{~mm}$ pellet. c) In the TEM, a Ta mesh grid, which also has catalyst particles dispersed over it, is placed above the pellet. Images are acquired from particles dispersed on the Ta mesh, while the entire sample contributes to the gas composition within the TEM.

Figure 2. Photographs of an actual sample pellet. a) Pellet with Ta mesh grid used for imaging. b) Pellet with no mesh. c) Same pellet as in b, but viewed edge-on to show the $0.65 \mathrm{~mm}$ thickness.
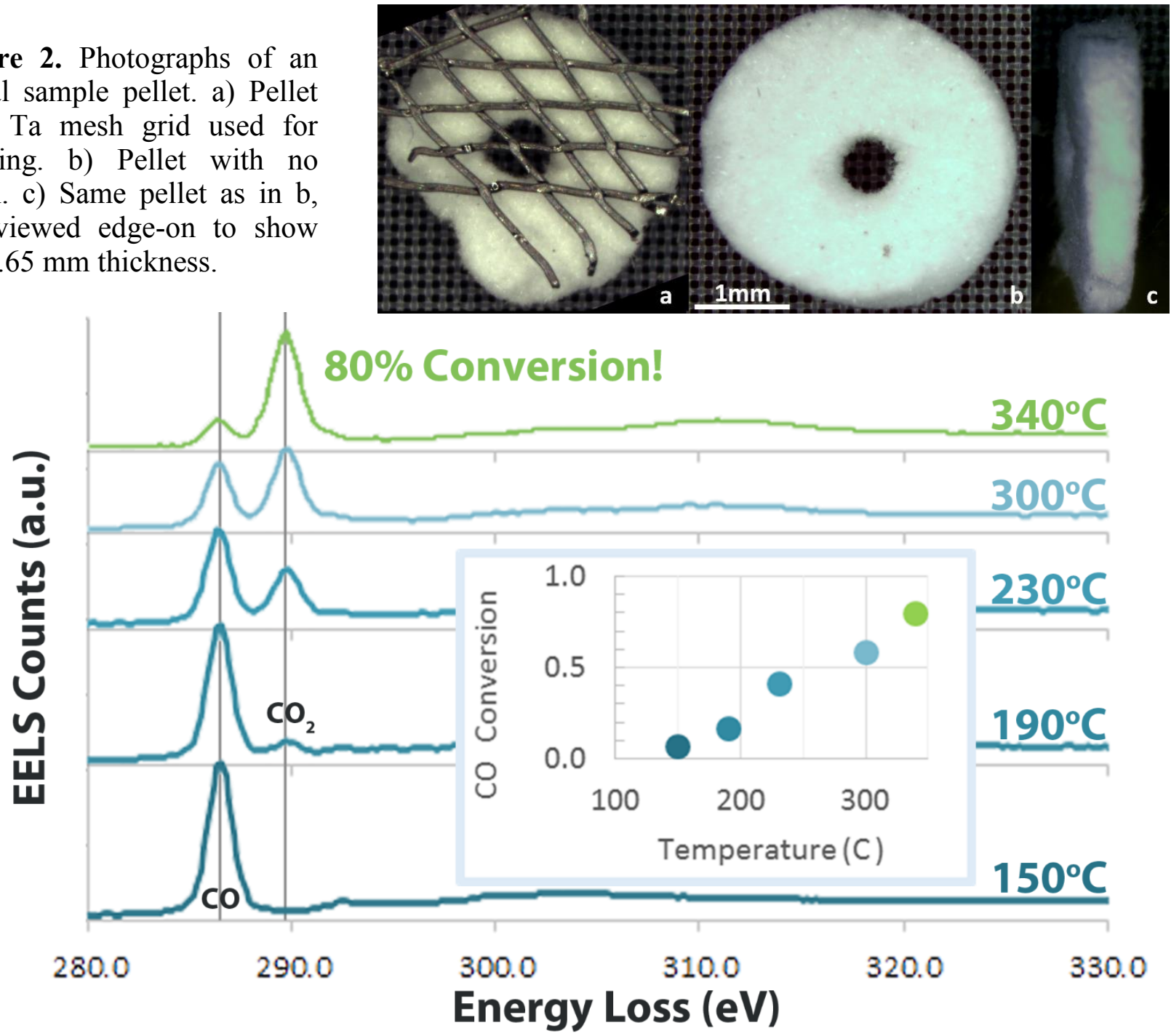

Figure 3. EELS spectra showing the conversion of $\mathrm{CO}$ to $\mathrm{CO} 2$ as the temperature of the pellet inside the microscope is increased. The inset shows CO conversion based on quantification of the EELS signals. 